\title{
Ultrafast Pump-Probe Spectroscopy of Finite-Sized Neutral Iron Oxide Clusters
}

Received 21th July 2020, Accepted

DOI: /

\author{
Jacob M. Garcia, ${ }^{a b}$ Ryan E. Shaffer ${ }^{a b}$ and Scott G. Sayres ${ }^{a b *}$
}

Neutral iron oxide clusters $\left(\mathrm{Fe}_{n} \mathrm{O}_{m}, \mathrm{n}, \mathrm{m} \leq 16\right)$ are produced in a laser vaporization source using $\mathrm{O}_{2}$ gas seeded in $\mathrm{He}$. The neutral clusters are ionized with a sequence of femtosecond laser pulses and detected using time-of-flight mass spectrometry. Small clusters are confirmed to be most prominant in the stoichiometric $(n=m)$ distribution, with $m=n+1$ clusters observed above $n=4$. Pump-probe spectroscopy is employed to study the dynamics of an electron transfer from an oxygen orbital to an iron nonbonding orbital of iron oxide clusters that is driven by absorption of a $400 \mathrm{~nm}$ photon. $\mathrm{A}$ bifurcation of the initial wavepacket occurs, where a femtosecond component is attributed to electron relaxation assisted through internuclear vibrational relaxation, and a slow relaxation shows the formation of a bound excited state. The lifetime and relative ratio of the two pathways depends on both the cluster size and iron oxidation state. The femtosecond lifetime decreases with increased cluster size until a saturation timescale is achieved at $n>5$. The relative population of the longlived excited state decreases with cluster size and suggests that the excited electron remains on the Fe atom for $>20$ ps.

\section{Introduction}

Iron oxides are important to several chemical processes such as corrosion and oxygen transport in biological systems. They serve as economical alternatives for replacing costly precious noble metals in industrial catalytic reactions because they exhibit good catalytic lifetimes as well as resistance to high concentrations of moisture and $\mathrm{CO}_{2}$, which often poison catalysts. Iron oxides are commonly used in the production of ammonia via the Haber-Bosh process, ${ }^{1}$ and hydrogen synthesis from the water-gas shift (WGS) reaction. ${ }^{2}$ Not only are iron oxides abundantly distributed around the earth's surface, iron monoxide is also important to the chemistry of the mesosphere. Further, iron oxide clusters are thought to play an important role in interstellar media, ${ }^{3-5}$ and are observed in the emission spectra of stars. ${ }^{6}$

Iron oxide clusters, which have been the subject of many gas phase studies due to their ease of production and ability to provide a molecular level understanding of bulk-phase catalytic mechanisms, have been shown to be highly active for a variety of industrially relevant chemical processes. Ionic clusters have been used for the oxidation of $\mathrm{CO},{ }^{7-9}$ methane, ${ }^{10}$ methanol, ${ }^{11,12}$ and various hydrocarbons. ${ }^{10,13}$ Neutral clusters have also shown activity for $\mathrm{CO}$ oxidation, ${ }^{14}$ methanol dehydrogentation, ${ }^{15}$ and oxidation of isoprene. ${ }^{16}$

\footnotetext{
a. School of Molecular Sciences, Arizona State University, Tempe, AZ 85287

b. Biodesign Center for Applied Structural Discovery, Arizona State University, Tempe, AZ 85287

*E-mail: Scott.Sayres@asu.edu

Electronic Supplementary Information (ESI) available: figure for the photon order power study; figure to show no transient dynamics observed in negative time; figure to show the fitting of the transient data for larger clusters.
}

In addition to chemical reactivity, many studies have also explored how the geometry, electronic structure, and stability of iron oxide clusters evolve with increasing cluster size. The ionic cluster distribution of iron oxides have been explored under a variety of laser ablation conditions, ${ }^{17-20}$ and neutral cluster distributions have been recorded using multiphoton ionization (MPI) and single photon ionization (SPI) with nanosecond duration laser pulses. ${ }^{21,22}$ Unlike bulk iron oxides, which are known in three stoichiometries $\left(\mathrm{FeO}, \mathrm{Fe}_{2} \mathrm{O}_{3}, \mathrm{Fe}_{3} \mathrm{O}_{4}\right)$, the gas phase clusters show a strong preference for the stoichiometric combination $(\mathrm{FeO})_{\mathrm{n}}$ below $\mathrm{n}=10$.

Additionally, the geometric and electronic structures of iron oxide clusters, determined by several quantum approaches, differ substantially from bulk structures. ${ }^{23-26}$ There is direct FeFe bonding in the clusters that is not observed in bulk FeO. Small stoichiometric clusters $\left((\mathrm{FeO})_{n}, \mathrm{n}<4\right)$ form highly stable monocyclic 2D ring structures, where each oxygen atom bridges two iron atoms. Computational studies suggest that larger clusters form through the assembly of these rings which stack to form layered structures, adopting a more compact spherical shape. ${ }^{23}$ However, multiple isomers have been observed with ion mobility mass spectrometry and attributed to both 2D and 3D structures. ${ }^{27}$ The vibrational spectra of many cationic iron oxide clusters have been explored with IR photodesorption spectroscopy to confirm structures. ${ }^{28-30}$ Structurally, no significant changes are calculated between the neutral and charged clusters leading to strong Franck-Condon overlap. ${ }^{24}$

The low-lying electronic states of $\mathrm{FeO}$ have been explored both theoretically ${ }^{31-33}$ and experimentally. ${ }^{34-36}$ Anion photoelectron spectroscopy has explored the evolution of the electronic states of iron monoxide $\left(\mathrm{Fe}_{\mathrm{n}} \mathrm{O}\right)$ clusters up to $\mathrm{n}=6,{ }^{37}$ as well as other stoichiometric compositions up to $\mathrm{Fe}_{4} \mathrm{O}_{6} \cdot{ }^{38,39}$ 
However, variations with respect to electronic structures, spin multiplicities, and the richness of accessible structural isomers has posed considerable theoretical challenges for understanding iron oxide clusters.

Quantitative information about bond energies has been obtained by studying the dissociation patterns of small iron oxide cluster cations using collision-induced dissociation (CID), ${ }^{40-43}$ thermal desorption, 44 and photodissociation experiments. ${ }^{45}$ These experiments have shown that the bridging oxygen atom in Fe-O-Fe is strongly bound, and that oxygen rich clusters contain $\mathrm{O}_{2}$ units with low binding energy. For example, the dissociation energies for oxygen rich clusters, containing up to six iron atoms have been measured showing $\mathrm{O}_{2}$ units are weakly bound $(<0.8 \mathrm{eV}) .{ }^{44}$ The per atom binding energy of stoichiometric iron oxide clusters is high ( $\sim .5 \mathrm{eV})$, independent of cluster size from $\mathrm{n}=2$ to $16 .^{23,46}$ These strong bonds require multiphoton absorption with $3.5 \mathrm{eV}$ photons for significant photodissociation. ${ }^{45}$ Due to the large binding energy, fragmentation is considered to be a local process, where clusters typically lose a single $\mathrm{FeO}$ or $\mathrm{O}_{2}$ unit to produce a stoichiometric cluster upon excitation.

Although the dissociation energies and chemical reactivity of small iron oxide clusters have been well characterized, timeresolved photoexcitation experiments of neutral iron oxides have not yet been reported. In addition, iron oxides have not been explored with femtosecond ( $f s$ ) laser pulses and the lifetimes of photoexcited states in small iron oxide nanoparticles or clusters are not well established. Here, we employ fs pump-probe spectroscopy to explore the relationship between the relaxation dynamics of iron oxide clusters and their size and composition. Patterns in the relaxation dynamics of clusters from the pump probe transients are presented. The ratio of amplitude coefficients between the fast $f s$ response and the longer picosecond response is utilized as a method to interpret the changes in the relaxation dynamics of iron oxide clusters due to size and stoichiometry. With the data presented herein, details are provided regarding the electronic relaxation timescales and stability of iron oxide clusters following photoexcitation.

\section{Experimental}

\subsection{Cluster Production and Detection}

Gas-phase cluster studies were performed using a homebuilt Wiley-McLaren ${ }^{47}$ type time-of-flight mass spectrometer (TOFMS) coupled to a fs laser system (Fig. 1). The second harmonic of a Nd:YAG laser (Spectra Physics Quanta-Ray INDI 50) was focused through a $25 \mathrm{~cm}$ lens for ablation of a $1 / 4$ " iron rod that was mechanically translated and rotated to provide a fresh target for every laser shot. A synchronized pulse of 1-20\% $\mathrm{O}_{2}$ seeded in helium carrier gas (100 psi) was introduced directly over the Fe rod. The plasma plume was confined to a $2 \times 60 \mathrm{~mm}$ collision cell to aid in cluster formation. The dense, hot plasma undergoes supersonic expansion and cooling to form clusters. After an additional $5 \mathrm{~cm}$ of travel, the plume traversed a $2 \mathrm{~mm}$ skimmer to form a collimated molecular beam. The ions

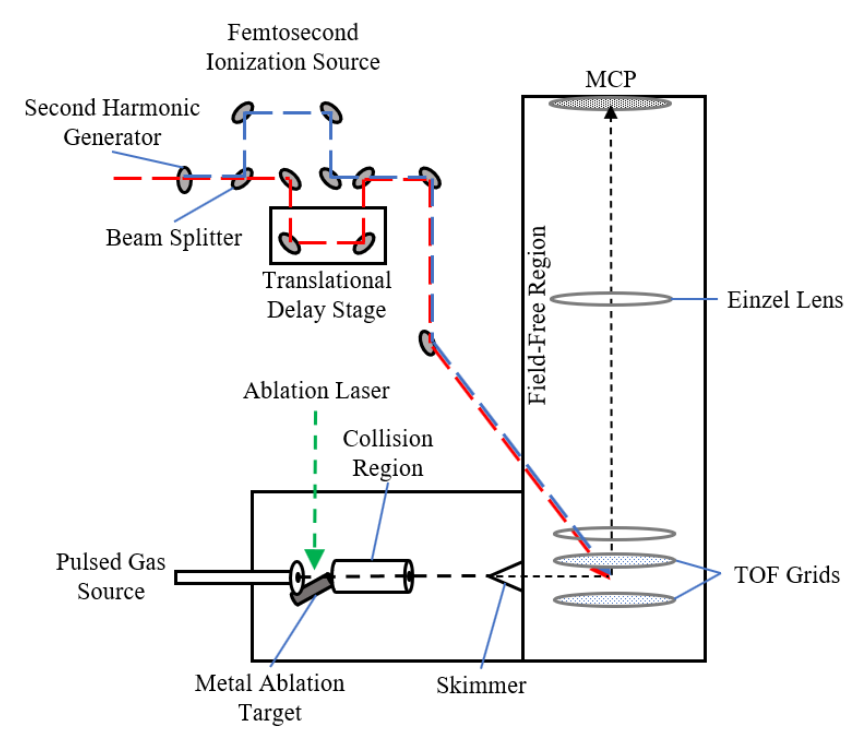

Fig. 1 Schematic of the gas phase cluster instrument and mass spectrometer. The fs laser beam path is shown.

produced through laser ablation were deflected by high voltage (200 V) placed on the skimmer, allowing only neutral clusters to enter the extraction region of the mass spectrometer. Neutral clusters were ionized through the interaction with a sequence of pulses from a Ti:Sapphire laser system (Spectra Physics Solstice Ace $1 \mathrm{kHz}$ rep. rate, $800 \mathrm{~nm}$ central wavelength).

lonized species were accelerated by a $10 \mu$ s high voltage ( 4 $\mathrm{kV})$ pulse on the TOF grids and steered toward the detector using an Einzel lens. The clusters were separated in arrival time through a 1.5-meter-long field-free region and detected by a chevron-type microchannel plate (MCP) detector. The observed signal was averaged for 1000 spectra, recorded by a digital oscilloscope, and transferred to a computer via a IEEE-488 interface for analysis. The vacuum pressure of $\sim 7.5 \times 10^{-8}$ Torr is maintained by three turbomolecular pumps. Synchronization of the laser pulses, gas valve, and TOF grids are controlled using a DG535 pulse generator. Spectra are sampled at $10 \mathrm{~Hz}$ in synchronization with the ablation source.

\subsection{Pump Probe Transients}

The ionization of the neutral cluster beam was performed by a series of $f$ laser pulses via a two-color pump-probe experiment. Briefly, $1 \mathrm{~mJ}$ of the $800 \mathrm{~nm}$ laser pulse was sent through a second harmonic doubling crystal (KDP crystal) to generate the $400 \mathrm{~nm}$ pump beam. The two beams are separated using a dichroic mirror and the $800 \mathrm{~nm}$ laser pulse travels across a programmable delay-stage with steps as short as $0.1 \mu \mathrm{m}$ before being recombined with the $400 \mathrm{~nm}$ beam using another dichroic mirror. The 400 and $800 \mathrm{~nm}$ laser beams are focused through a $50 \mathrm{~cm}$ lens to reach intensities of up to $8.5 \times 10^{14}$ and $2.4 \times 10^{15} \mathrm{~W} / \mathrm{cm}^{2}$, respectively. Pump-probe transients were recorded by integrating the ion signal over an average of 350 spectra at each time delay as the $800 \mathrm{~nm}$ beam was scanned from -2 ps to +20 ps in $40 \mathrm{fs}$ increments. The recorded dynamics 
were not affected by changes in the oxygen concentration between $1-20 \%$.

\subsection{Signal Analysis}

We consider the bifurcation of the initial wavepacket, requiring two fitting functions to account for the transient ion signal. The transient signals for each ion are analyzed by fitting a combination of an exponential decay and plateau function to the data using a least squares method that has been described in detail elsewhere. ${ }^{48}$ The exponential decay function is convoluted with the Gaussian instrumental response function and accounts for the fast relaxation in transient signals associated with an intermediate metastable state of a neutral species that decays with the measured lifetime $(\tau)$. A plateau function is included to account for excited states with lifetimes longer than timescales addressed in the experiment. The relative contributions of the scaling coefficients of exponential $\left(C_{E}\right)$ and plateau $\left(C_{P}\right)$ fitting functions are used to interpret the cluster signals. Cluster dissociation is typically accompanied by a fs increase in ion signal following the pump pulse excitation, thereby requiring an exponential growth function for fitting. This growth is only observed in $\mathrm{Fe}_{2}{ }^{+}$which will be described below. Therefore, the dissociation channels contribute a negligible amount to the ion transient signals.

The $800 \mathrm{~nm}$ beam was measured through autocorrelation to be $<35 \mathrm{fs}$. The instrumental response (Gaussian function), measured through a cross-correlation of the $400 \mathrm{~nm}$ pump and $800 \mathrm{~nm}$ probe beams over the non-resonant ionization of $\mathrm{O}_{2}$ directly in the molecular beam was measured to be $<60 \mathrm{fs}$ (FWHM). The maximum intensity of the cluster signals is recorded $\sim 50 \mathrm{fs}$ after the temporal overlap of the two laser pulses (time zero). The transient ion signal is a convolution of the molecular response and the cross-correlation of the two laser beams and therefore the maximum of the ion signal exhibits a temporal shift proportional to the lifetime of the cluster.

\section{Results \& Discussion}

Dissociation from cation states was minimized by applying only threshold laser intensities for ionization. At negative probe delay, the $800 \mathrm{~nm}$ beam interacts with the clusters prior to the $400 \mathrm{~nm}$ beam and no discernable dynamics are observed The signal at negative times matches the sum of the ion signal produced by the individual 800 and $400 \mathrm{~nm}$ beams (supporting information, Fig. S1). Therefore, the relaxation of the $800 \mathrm{~nm}$ photon absorption is rapid and subsequent absorption from the UV beam is insufficient for ionization. Small fluctuations in signal are attributed to inconsistencies in laser ablation conditions.

The IP of $\mathrm{FeO}$ is $8.56 \mathrm{eV}, 49$ requiring the absorption of one $400 \mathrm{~nm}$ and four $800 \mathrm{~nm}$ photons for ionization. After ionization, the cation contains up to $0.74 \mathrm{eV}$ of energy above the ground state, and is stable by remaining below the dissociation limit of $\mathrm{FeO}^{+}(3.52 \pm 0.02 \mathrm{eV}) .{ }^{49}$ This photon dependence in our experiments is supported by a power study

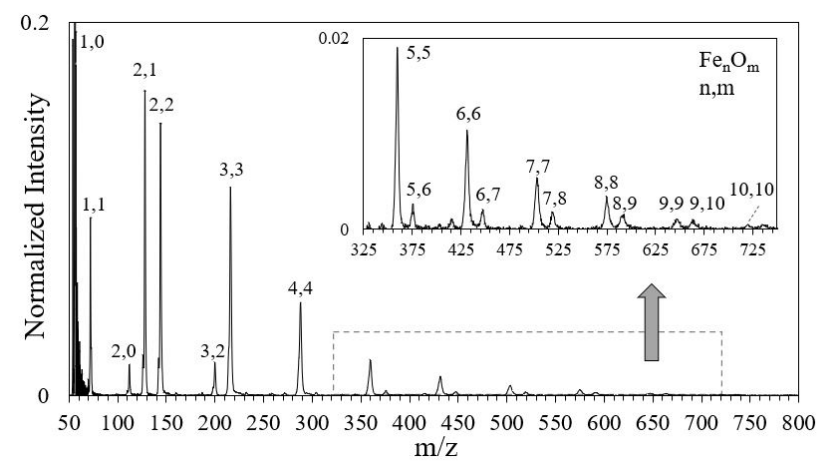

Fig. 2 Mass spectrum of neutral iron oxide clusters produced with $10 \% \mathrm{O} 2$ seeded in $\mathrm{He}$, recorded at temporal overlap of the pump and probe beams. The mass spectrum is normalized to the largest signal intensity $\left(\mathrm{Fe}^{+}\right)$and averaged over 1000 laser shots.

(supporting information, Fig. S2). The combined photon energies of the pump and four probe laser beams $(9.3 \mathrm{eV})$ exceeds the IP for all clusters, with the IPs of $(\mathrm{FeO})_{2-5}$ calculated to be $7.46,7.59,7.39$, and $7.60 \mathrm{eV}$, respectively. ${ }^{23}$ Therefore, the absorption of one pump and four probe photon by each of the $(\mathrm{FeO})_{2-5}$ clusters leaves an excess of $1.84,1.71,1.91$, and $1.70 \mathrm{eV}$ above the cation ground states, respectively, and remains below the dissociation energies. For the long-lived states of these clusters to be ionized with four probe photons, the wavepacket must remain at least $1.16,1.29,1.09$, and 1.3 $\mathrm{eV}$ above the ground states of $(\mathrm{FeO})_{2-5}$. Although the IPs of larger cluster have not yet been reported, they are predicted to decrease slightly with size. ${ }^{21}$

\section{1. $\mathrm{Fe}_{n} \mathrm{O}_{\mathrm{m}}$ Cluster Distribution}

The iron oxide cluster distribution recorded in the mass spectrometer depends on several experimental parameters such as ablation laser power, relative timing of the nozzle expansion, oxygen concentration, and backing gas pressure. The cation distribution produced via the fs laser ionization of neutral clusters (Fig. 2) is generally consistent with previous photoionization mass spectra of neutral $\mathrm{Fe}_{n} \mathrm{O}_{m}$ beams, ${ }^{21,22}$ and cations generated directly from laser vaporization sources. ${ }^{45}$ However, single photon ionization with vacuum ultraviolet (VUV) lasers may be free of fragmentation, allowing weakly bound oxygen rich clusters to be observed from the ionization of neutral clusters. ${ }^{14-16,21}$ Oxygen rich clusters are typically small signals in the cluster distribution. Even at high oxygen concentrations (up to $20 \%$ ), oxygen rich cation clusters, such as $\mathrm{Fe}_{2} \mathrm{O}_{\mathrm{m}}{ }^{+}$with $\mathrm{m}>2$, are not observed in our mass spectra using fs photoionization. We suspect that if oxygen rich clusters were present in the neutral distribution, they likely dissociate during or after the ionization process due to low binding energies. For example, oxygen rich cation clusters have low thresholds for $\mathrm{O}_{2}$ dissociation $\left(<0.7 \mathrm{eV}^{43}\right)$ for the $\mathrm{Fe}_{2}$ series, except for $\mathrm{Fe}_{2} \mathrm{O}_{3}{ }^{+}$ which has a threshold energy of $1.14 \mathrm{eV} .{ }^{44} \mathrm{Fe}_{2} \mathrm{O}_{2}{ }^{+}$and $\mathrm{Fe}_{2} \mathrm{O}^{+}$, which are observed here have dissociation energies exceeding $4 \mathrm{eV}$ and $5 \mathrm{eV},{ }^{43}$ respectively.

The intensities of the $(\mathrm{FeO})_{n}$ ions are plotted as a function of size in Fig. 3. Above $n=3$, the relative intensities of the $(\mathrm{FeO})_{n}{ }^{+}$ 


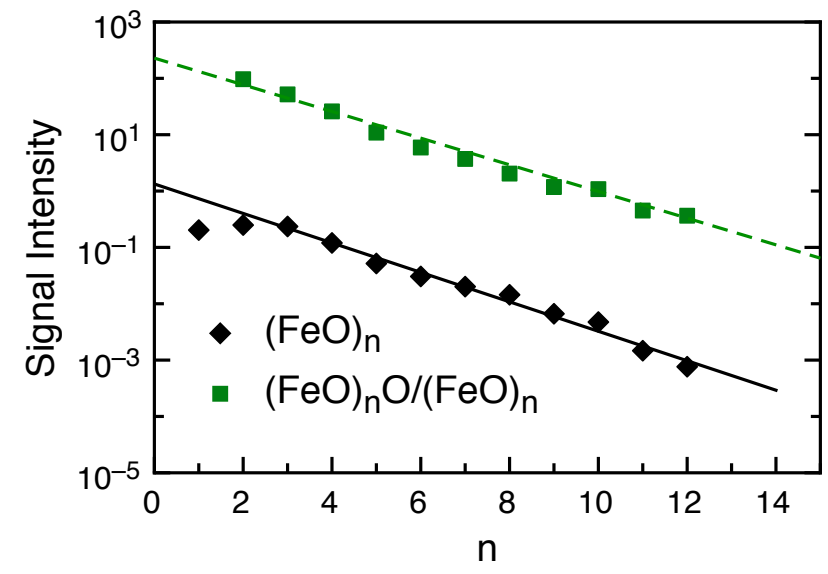

Fig. 3 The integrated ion signal intensities of iron oxide neutral clusters produced under $10 \% \mathrm{O}_{2}$ seeded in $\mathrm{He}$. The signal intensity of $(\mathrm{FeO})_{n}$ (black diamonds, solid line) and $(\mathrm{FeO})_{n} \mathrm{O} /(\mathrm{FeO})_{n}$ (green squares, dashed line) is plotted on a logarithmic scale.

clusters decay exponentially with the addition of each FeO unit. This is consistent with a typical thermodynamic cluster distribution and suggests that the ions accurately represent the neutral distribution. The intensity of the $\mathrm{Fe}_{n} \mathrm{O}_{n+1}{ }^{+}$series compared to the $(\mathrm{FeO})_{n}{ }^{+}$series is also plotted as a function of size in Fig. 3. The $\mathrm{Fe}_{n} \mathrm{O}_{n+1}$ cluster series has significant intensity above $\mathrm{n}=5$ and exceeds the signal intensity of the stoichiometric species above $\mathrm{n}=10$, where the binding energy of an additional $\mathrm{O}$ atom becomes comparable to that of an $\mathrm{FeO}$ unit. In our experiment, the largest cluster observed is $\mathrm{Fe}_{15} \mathrm{O}_{16}{ }^{+}$ (not shown). The $\mathrm{Fe}_{3} \mathrm{O}_{\mathrm{m}}$ and $\mathrm{Fe}_{2} \mathrm{O}_{\mathrm{m}}$ series both contain strong signals corresponding to suboxide stoichiometries and will be discussed below.

The per atom binding energies of iron oxide neutral clusters are high ( $\sim .5 \mathrm{eV}$ ) from $\mathrm{n}=2$ through $\mathrm{n}=16,23,46$ and increases to $>5 \mathrm{eV}$ for the cations. ${ }^{24}$ The binding energy of FeO units are also high $(\sim 4 \mathrm{eV})$ for neutral $(\mathrm{FeO})_{\mathrm{n}}$ clusters. ${ }^{46}$ Due to the high bond energies, and since the dissociation thresholds of most iron oxide clusters are above the photon energy from either the pump or probe pulse, we rule out fragmentation as a major ion source. Thus, most ions recorded in the mass spectra are attributed to either direct ionization, or as products from the loss of weakly bound $\mathrm{O}_{2}$ units that do not significantly affect the transient dynamics.

\subsection{The $(\mathrm{FeO})_{\mathrm{n}}$ Series Dynamics}

The transient dynamics of the stoichiometric $(\mathrm{FeO})_{n}$ cluster series are presented in Fig. 4. The increase in the temporal profile of the clusters indicates that a state, or band of states, with an appreciable lifetime is being accessed by the pump laser. Two distinct relaxation pathways are observed in the transient signals and will be described separately.

The fs lifetime of the $(\mathrm{FeO})_{n}{ }^{+}$transients is plotted against their cluster size (Fig. 5) and shows that the excited state lifetimes of the clusters decreases as they grow in size. Since the stoichiometric clusters are strongly bound, the fs relaxation timescale is likely too short to be attributable to a fragmentation event, and instead is related to the timescale of intramolecular vibrational redistribution (IVR) that assists in returning the excited electron back to the ground state. The decreasing lifetime of the clusters as more FeO units are incorporated shows that the fs relaxation component is assisted by the larger cluster's additional vibrational degrees of freedom. The excited state lifetime decreases steadily from $n=$

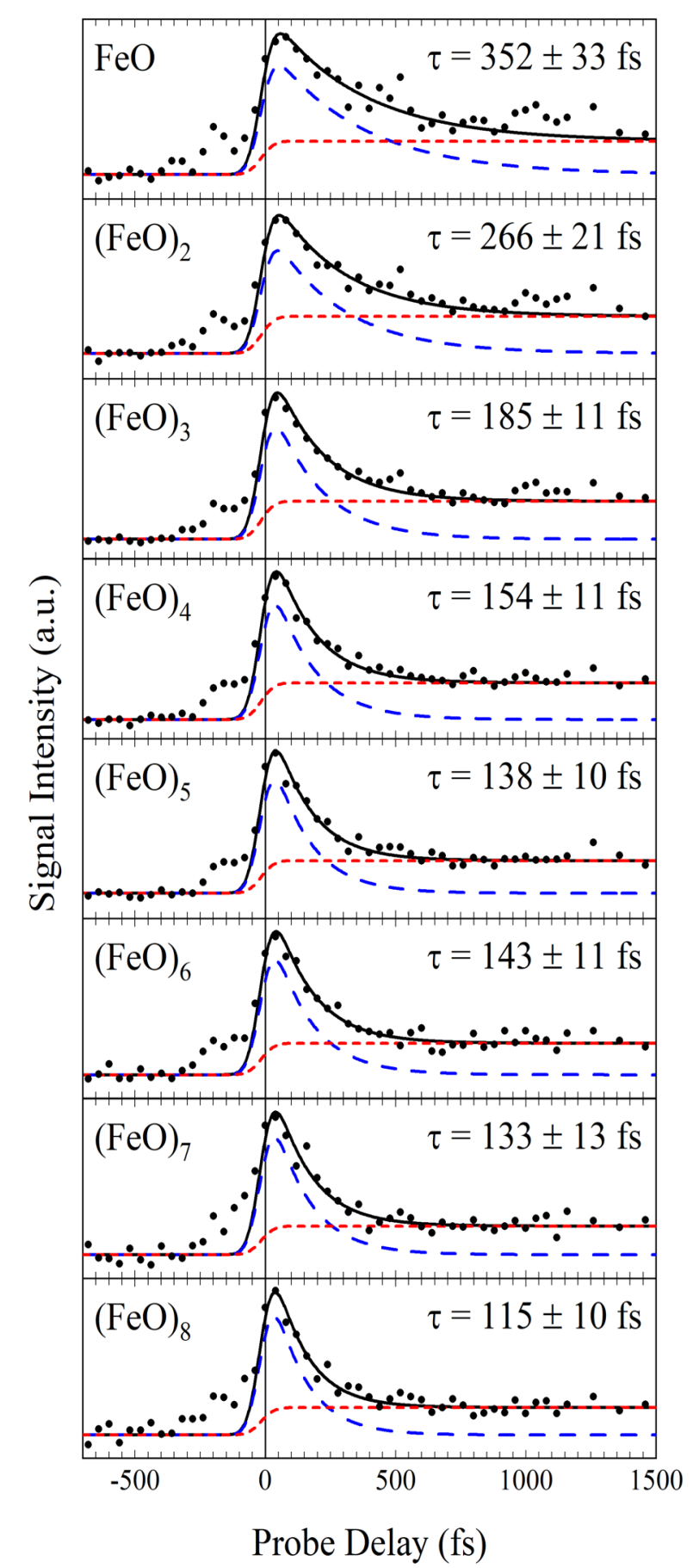

Fig. 4 The pump-probe transients of the $(\mathrm{FeO})_{n}$ series from $n=1-12$ with the separate fitting functions shown as the exponential decay function (blue long dash), and plateau function (red short dash) making the total fit (black solid). The time points are spaced by $40 \mathrm{fs}$ out to $1100 \mathrm{fs}$, then by $100 \mathrm{fs}$ out to $20 \mathrm{ps}$, but truncated to highlight the fast response. A vertical black line appears at the temporal overlap of the laser beams. 


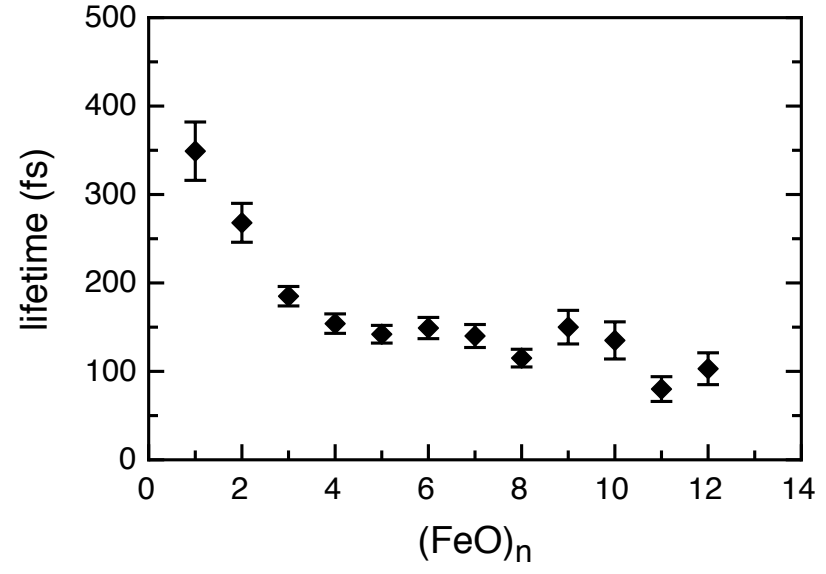

Fig. 5 The relaxation lifetime as a function of cluster size in the $(\mathrm{FeO})$ n cluster series.

1 , which has a lifetime of $352 \pm 33 \mathrm{fs}$, before reaching a consistent value for clusters larger than $n=5$. Above $n=5$, the relaxation dynamics ( $\tau=103-143 \mathrm{fs}$ ) are a factor of $\sim 2.5 \mathrm{x}$ faster than in $\mathrm{FeO}$. This saturation in lifetime coincides with the cluster size known to be the onset of 3D cluster geometries. The fitting coefficients and relaxation times of the $(\mathrm{FeO})_{n}(n<13)$ series are summarized in Table 1.

Stoichiometric clusters have a similar transient signal, suggesting a common chromophore is being accessed. The electronic structure of $\mathrm{FeO}$ is well understood and serves as a starting point to describe the nature of the larger cluster's excited states prepared by the absorption of a $400 \mathrm{~nm}$ photon. The manifold of excited states for $\mathrm{FeO}$ is dense, ${ }^{32}$ having 49 states within an energy range of $3.1 \mathrm{eV} .{ }^{32}$ Several low-lying bands have been well characterized below the dissociation limit of $4.20 \pm 0.13 \mathrm{eV} .50$ The ground state molecular orbital configuration of $\mathrm{FeO}$ is $8 \sigma^{2} 3 \pi^{4} 4 \pi^{2} 1 \delta^{39} \sigma^{1}$, where the O-derived orbitals lie below the Fe 3 d-derived orbitals, formally resulting in a charge transfer of two electrons from the Fe to $\mathrm{O}$ atom. The lower lying electronic $A$ and $B / C$ states recorded in photoelectron spectroscopy are attributed to excitation from the Fe $3 \mathrm{~d}$ orbitals ( $1 \delta$ or $9 \sigma$ ). ${ }^{39}$ The $\mathrm{D}$ state resides at $1.89 \mathrm{eV}$ and is attributed to excitation of the $4 \pi$ electron, which is also from the Fe $3 \mathrm{~d}$ orbital. ${ }^{39}$ The excited state accessed here, with $3.1 \mathrm{eV}$, arises from excitation of either the $3 \pi$ or $8 \sigma$ orbitals which have predominantly oxygen character. Thus, the excited electron moves from the low-lying orbitals that are predominantly oxygen to a nonbonding Fe orbital.

Photoelectron spectroscopy performed on iron monoxides has shown only small changes in energy levels with increasing iron atoms. ${ }^{37}$ The strong similarities with the photoelectron spectra of $\mathrm{FeO}^{35,39}$ enables us to apply the spectra of the larger clusters to approximate the electronic structure of FeO. The larger $(\mathrm{FeO})_{n}$ clusters all present higher energy states following a $\sim 1$ eV gap above the Fe orbitals, where several less characterized states appear. A broad band is observed at $\sim 3 \mathrm{eV}$ above the ground state that is assigned as the $\mathrm{G}$ band in $\mathrm{Fe}_{2} \mathrm{O} .{ }^{37}$ Thus, we tentatively assign the excitation of the stoichiometric clusters as $\mathrm{G}$ band excitations.
Table 1. Fitting coefficients for the ultrafast ionization of stoichiometric iron oxide clusters.

\begin{tabular}{lllll}
\hline Species & $C_{E}$ & $\tau(\mathrm{fs})$ & $C_{P}$ & $V$ \\
\hline $\mathrm{FeO}$ & 1.010 & $352 \pm 33$ & 0.239 & 0.237 \\
$\mathrm{Fe}_{2} \mathrm{O}_{2}$ & 1.042 & $266 \pm 21$ & 0.273 & 0.262 \\
$\mathrm{Fe}_{3} \mathrm{O}_{3}$ & 1.175 & $185 \pm 11$ & 0.267 & 0.228 \\
$\mathrm{Fe}_{4} \mathrm{O}_{4}$ & 1.251 & $154 \pm 11$ & 0.252 & 0.201 \\
$\mathrm{Fe}_{5} \mathrm{O}_{5}$ & 1.309 & $138 \pm 10$ & 0.232 & 0.177 \\
$\mathrm{Fe}_{6} \mathrm{O}_{6}$ & 1.341 & $143 \pm 11$ & 0.218 & 0.163 \\
$\mathrm{Fe}_{7} \mathrm{O}_{7}$ & 1.502 & $133 \pm 13$ & 0.204 & 0.136 \\
$\mathrm{Fe}_{8} \mathrm{O}_{8}$ & 1.563 & $115 \pm 10$ & 0.187 & 0.119 \\
$\mathrm{Fe}_{9} \mathrm{O}_{9}$ & 1.390 & $150 \pm 19$ & 0.159 & 0.114 \\
$\mathrm{Fe}_{10} \mathrm{O}_{10}$ & 1.447 & $135 \pm 21$ & 0.139 & 0.096 \\
$\mathrm{Fe}_{11} \mathrm{O}_{11}$ & 1.601 & $80 \pm 14$ & 0.154 & 0.096 \\
$\mathrm{Fe}_{12} \mathrm{O}_{12}$ & 1.622 & $103 \pm 18$ & 0.149 & 0.092 \\
\hline
\end{tabular}

A similar argument can be applied to larger stoichiometric clusters, although their electronic structures are less known. The excited states of the clusters become more congested and the density of states increases with size. Even clusters as small as $\mathrm{Fe}_{3} \mathrm{O}_{3}$ and $\mathrm{Fe}_{4} \mathrm{O}_{4}$ exhibit no sharp peaks in photoelectron studies, suggesting a high density of states. ${ }^{38}$ Although assignments of the excited states are difficult, they also arise through transfer of an electron from the $\mathrm{O}$ atom to the Fe atom.

The ratio between the fitting coefficients, $Y\left(C_{P} / C_{E}\right)$, decreases linearly with cluster sizes above $n=2$ for the stoichiometric clusters. For $\mathrm{FeO}, \sim 25 \%$ of the population remains in the excited state for $>20$ ps. The excitation photon energy remains below the dissociation energy, and therefore the long decay is a consequence of populating a bound state. Four probe photons can ionize an excited neutral state that is at least $2.26 \mathrm{eV}$ above the ground state. Therefore, neither the $A$ state nor the $B / C$ states, which are $\sim 0.5 \mathrm{eV}$ and $\sim 1 \mathrm{eV}$ above the ground state, accounts for the long-lived signal. This suggests that the excited electron does not transfer back to the oxygen atom within the $20 \mathrm{ps}$ timescale. The $\gamma$ for $\mathrm{FeO}$ is 0.237 , increases to 0.262 for $n=2$, and then decreases almost linearly with the addition of each FeO unit. The increased density of states for larger clusters enables a larger proportion of the wavepacket to undergo electronic relaxation to low energy states that are inaccessible for ionization by the probe pulse.

\section{3 $\mathrm{Fe}_{2} \mathrm{O}_{m}$ Dynamics}

Changes in the ultrafast relaxation dynamics due to oxidation state are observed by comparing the dynamics of clusters containing the same number of metal atoms but varying number of oxygen atoms. If we assume that each $O$ atom has an oxidation state of -2 , then the oxidation state of the $\mathrm{Fe}$ atoms can be evaluated in each cluster such that in neutral $(\mathrm{FeO})_{\mathrm{n}}$ all the $\mathrm{Fe}$ atoms have $\mathrm{a}+2$ oxidation state. For $\mathrm{Fe}_{2} \mathrm{O}$, where the $\mathrm{Fe}$ atoms share the $\mathrm{O}$ atom equally, the oxidation state of each of the Fe atoms is +1 .

The excited state lifetime decreases with additional oxygen atoms, and therefore shows that the higher oxidation state of 
Table 2. Fitting coefficients for the ultrafast ionization of selected mid-range iron oxide clusters.

\begin{tabular}{lllcc} 
Species & $C_{E}$ & $\tau(\mathrm{fs})$ & $C_{P}$ & $V$ \\
\hline $\mathrm{Fe}_{2}$ & 0.586 & $699 \pm 163$ & 0.272 & 0.464 \\
$\mathrm{Fe}_{2} \mathrm{O}$ & 0.883 & $404 \pm 37$ & 0.299 & 0.339 \\
$\mathrm{Fe}_{3} \mathrm{O}$ & 1.000 & $526 \pm 104$ & 0.000 & 0.000 \\
$\mathrm{Fe}_{3} \mathrm{O}_{2}$ & 0.988 & $323 \pm 43$ & 0.301 & 0.305 \\
$\mathrm{Fe}_{3} \mathrm{O}_{4}$ & 1.708 & $85 \pm 20$ & 0.187 & 0.110 \\
$\mathrm{Fe}_{5} \mathrm{O}_{6}$ & 1.696 & $116 \pm 21$ & 0.154 & 0.091 \\
$\mathrm{Fe}_{6} \mathrm{O}_{5}$ & 1.235 & $158 \pm 32$ & 0.160 & 0.130 \\
$\mathrm{Fe}_{6} \mathrm{O}_{7}$ & 2.029 & $68 \pm 10$ & 0.099 & 0.049 \\
\hline
\end{tabular}

iron leads to faster electron relaxation back to the oxygen. The fs lifetimes of $\mathrm{Fe}_{2} \mathrm{O}_{2}, \mathrm{Fe}_{2} \mathrm{O}$, and $\mathrm{Fe}_{2}$ are shown in Fig. 6 to be 268 $\pm 22 \mathrm{fs}, 404 \pm 37 \mathrm{fs}$, and $699 \pm 163 \mathrm{fs}$, respectively. Further, the $\gamma$ values for the $\mathrm{Fe}_{2} \mathrm{O}_{\mathrm{m}}{ }^{+}$series decreases from 0.464 to 0.262 with the addition of oxygen atoms. Therefore, with the increase in oxidation state of the $\mathrm{Fe}$, less of the population remains in the bound excited electronic state. The fitting coefficients for $\mathrm{Fe}_{2} \mathrm{O}_{\mathrm{m}}{ }^{+}$and the other non-stoichiometric clusters $(n<7)$ are summarized in Table 2.

The $\mathrm{Fe}_{2} \mathrm{O}_{\mathrm{m}}$ cluster series is the most studied series of iron oxide clusters, and the nature of the excited state of $\mathrm{Fe}_{2} \mathrm{O}$ can be assigned using data provided by photoelectron spectra. A broad band is observed at $\sim 3 \mathrm{eV}$ above the ground state that is assigned as the $\mathrm{G}$ band in $\mathrm{Fe}_{2} \mathrm{O} .{ }^{37} \mathrm{Fe}_{2} \mathrm{O}$ has an IP of $6.78 \pm 0.05$ $\mathrm{eV}$ and remains just below the $\left(\mathrm{FeO}^{+}+\mathrm{Fe}\right)$ dissociation energy of $2.60 \mathrm{eV}$ upon excitation with the $1+4$ pulse sequence. ${ }^{37}$

The maximum signal intensity for the $\mathrm{Fe}_{2} \mathrm{O}_{m}$ cluster series shifts in time delay with the number of oxygen atoms bound within the cluster (Fig. 6). $\mathrm{Fe}_{2}{ }^{+}$reaches its maximum signals at later time delays than the other clusters. However, the transient signal for $\mathrm{Fe}_{2}{ }^{+}$requires a growth function, with a lifetime $\tau_{\mathrm{g}}=40$ $\pm 15 \mathrm{fs}$, to accurately account for the dynamics. This growth is sufficiently fast to be attributed to direct dissociation of a larger

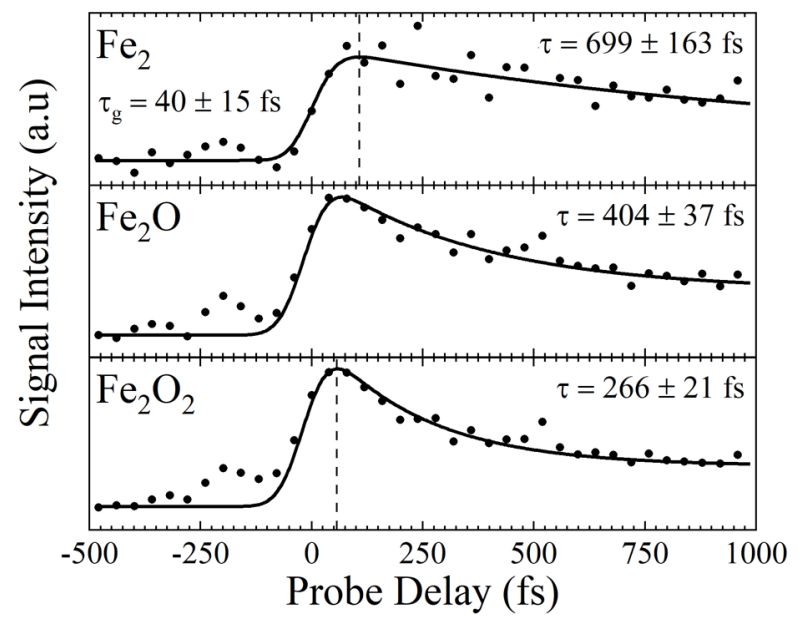

Fig. 6 Transients of $\mathrm{Fe}_{2} \mathrm{O}_{m}{ }^{+}(m \leq 2)$ showing a vertical dashed line drawn at the maximum of the fit signal for the clusters.

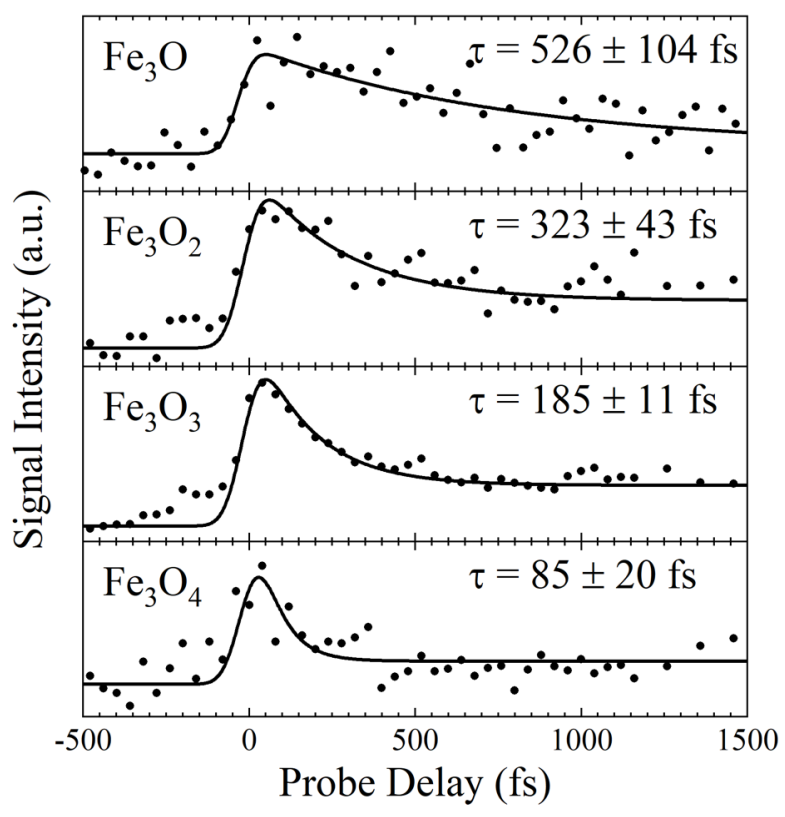

Fig. 7 Transient signals of $\mathrm{Fe}_{3} \mathrm{O}_{1-4^{+}}$clusters shown with total fits.

neutral cluster's excited state. $\mathrm{Fe}_{2}{ }^{+}$has a low IP $(6.3 \mathrm{eV})$ and can be ionized directly by four probe photons. The delay in ion signal suggests that $\mathrm{Fe}_{2}$ is not present in the neutral cluster beam, and instead highlights the dissociation dynamics of a larger neutral cluster after being exposed to the pump beam. Although we cannot identify the parent cluster from these experiments, one possibility is the facile dissociation of energetic isomers. For example, formation of $\mathrm{Fe}_{2}{ }^{+}$occurs at $\sim 0.5 \mathrm{eV}$ from $\mathrm{Fe}_{2} \mathrm{O}_{2}{ }^{+}$, attributed to a high energy isomer of $\mathrm{Fe}_{2} \mathrm{O}_{2} .{ }^{41}$

\section{4 $\mathrm{Fe}_{3} \mathrm{O}_{\mathrm{m}}$ Series Dynamics}

Several ion transients are recorded in the $\mathrm{Fe}_{3} \mathrm{O}_{m}(m=1-4)$ series (Fig. 7). $\mathrm{Fe}_{3} \mathrm{O}_{3}$ and $\mathrm{Fe}_{3} \mathrm{O}_{2}$ appear with strong signals, while $\mathrm{Fe}_{3} \mathrm{O}$ and $\mathrm{Fe}_{3} \mathrm{O}_{4}$ are weak signals. $\mathrm{Fe}_{3} \mathrm{O}_{m}(\mathrm{~m}>4)$ clusters have a $\mathrm{O}_{2}$ dissociative energy of $<0.6 \mathrm{eV}^{44}$ and are not expected to survive the ionization mechanism. Similar to the pattern described in the $\mathrm{Fe}_{2} \mathrm{O}_{\mathrm{m}}{ }^{+}$clusters, the lifetime of $\mathrm{Fe}_{3} \mathrm{O}_{\mathrm{m}}{ }^{+}$clusters also decrease with additional oxygen content. The lifetime of $\mathrm{Fe}_{3} \mathrm{O}_{3}{ }^{+}(185 \pm 11 \mathrm{fs})$ is shorter than $\mathrm{Fe}_{3} \mathrm{O}_{2}{ }^{+}(323 \pm 43)$, and $\mathrm{Fe}_{3} \mathrm{O}_{4}{ }^{+}$ exhibits the shortest lifetime of the series ( $85 \pm 20 \mathrm{fs})$. $\mathrm{Fe}_{3} \mathrm{O}^{+}$is only observed under very low oxygen concentrations, but also fits into this pattern with a long lifetime of $526 \pm 104 \mathrm{fs}$.

With a higher Fe content, the $\mathrm{O}$ atoms are no longer shared equally among the $\mathrm{Fe}$ atoms as they were for the $\mathrm{Fe}_{2} \mathrm{O}_{\mathrm{m}}$ series. For $\mathrm{Fe}_{3} \mathrm{O}_{2}$, one of the $\mathrm{Fe}$ atoms is attached to two oxygen atoms for a +2 oxidation state, and two of the Fe atoms only bind to one oxygen to have +1 oxidation states. $\mathrm{For} \mathrm{Fe}_{3} \mathrm{O}$, two of the $\mathrm{Fe}$ atoms are in $\mathrm{a}+1$ oxidation state and one $\mathrm{Fe}$ has an oxidation state of 0 . $\mathrm{Fe}_{3} \mathrm{O}_{4}$ has a structure similar to the $\mathrm{Fe}_{3} \mathrm{O}_{3}$, but contains an extra $\mathrm{O}$ bound to one of the terminal $\mathrm{Fe}$ atoms, making it a +4 oxidation state. Similar to the $\mathrm{Fe}_{2}$ series, removing one oxygen extends the lifetime of the state by 150 $\mathrm{fs}$, and removal of the second oxygen atom extends the lifetime 


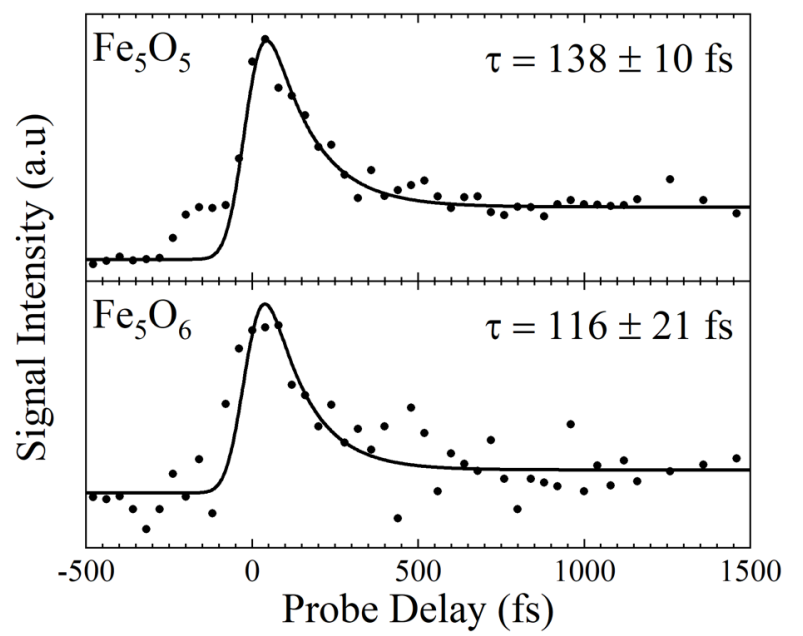

Fig. 8 Transient signal of $\mathrm{Fe}_{5} \mathrm{O}_{m}{ }^{+}(m=5-6)$ signals.

by $\sim 350 \mathrm{fs}$. This supports the argument that the lower oxidation state of the $\mathrm{Fe}$ atom extends the fs lifetime of the cluster transient. With the decrease in oxidation state, the $\psi$ values for the $\mathrm{Fe}_{3} \mathrm{O}_{\mathrm{m}}{ }^{+}$series also decreases, with the exception of $\mathrm{Fe}_{3} \mathrm{O}$ which is recorded with $\mathrm{a} \gamma=0.0 . \mathrm{Fe}_{3} \mathrm{O}_{2}$ has $\mathrm{a} \gamma=0.305$ and drops steadily with the addition of each oxygen until reaching 0.110 for $\mathrm{Fe}_{3} \mathrm{O}_{4}$. This decrease in $\gamma$ with oxygen units is consistent with $\mathrm{Fe}_{2} \mathrm{O}_{\mathrm{m}}$ clusters.

\section{5 $\mathrm{Fe}_{4} \mathrm{O}_{m}, \mathrm{Fe}_{5} \mathrm{O}_{m}$, and $\mathrm{Fe}_{6} \mathrm{O}_{m}$ Series Dynamics}

The lifetimes and patterns measured in the dynamics for the cluster series of $\mathrm{Fe}_{4} \mathrm{O}_{m}, \mathrm{Fe}_{5} \mathrm{O}_{m}$, and $\mathrm{Fe}_{6} \mathrm{O}_{m}$ are similar to those determined in smaller clusters. The stoichiometric clusters all have lifetimes of $\sim 140 \mathrm{fs}$. These mid-range clusters represent the transition in geometry from $2 \mathrm{D}$ rings to $3 \mathrm{D}$ structures. Some calculations have predicted that $\mathrm{Fe}_{5} \mathrm{O}_{5}$ is planar, ${ }^{23}$ while others have shown the 3D geometry is lower in energy. ${ }^{24}$ Overall, the 3D structures support a wider range of stable stoichiometries and enables stronger bonding arrangements with an extra oxygen atom. In $\mathrm{Fe}_{n} \mathrm{O}_{n+1}$, more Fe d electrons transfer to the additional $\mathrm{O}$ atom. The extra oxygen atom is likely bridged between two Fe atoms and therefore increases the oxidation state of two Fe atoms to +3 while the rest remain at +2 . This change in oxidation state will be most profound in smaller clusters, where a single oxygen makes the biggest adjustment to the overall cluster stoichiometry.

The $\mathrm{Fe}_{4}$ cluster series contains only one dominant cluster, $\mathrm{Fe}_{4} \mathrm{O}_{4}{ }^{+}$. The $\mathrm{Fe}_{5} \mathrm{O}_{m}(\mathrm{~m}=5-6)$ cluster series (Fig. 8) shows the presence of two clusters and is consistent with all other series, showing a $\mathrm{Fe}_{5} \mathrm{O}_{6}{ }^{+}$lifetime $(116 \pm 21 \mathrm{fs})$ slightly shorter than the $\mathrm{Fe}_{5} \mathrm{O}_{5}{ }^{+}$transient $(142 \pm 10 \mathrm{fs})$. Also, the $\gamma$ of $\mathrm{Fe}_{5} \mathrm{O}_{5}{ }^{+}$decreases from 0.177 to 0.091 with the additional oxygen atom. The ultrafast dynamics of the $\mathrm{Fe}_{6} \mathrm{O}_{m}(m=5-7)$ cluster series is shown in Fig. 9, and the trends are consistent with the smaller clusters. However, the effect of the missing oxygen on the relaxation dynamics is not as pronounced as in smaller clusters, and only extends the lifetime by $\sim 6 \%$ for $\mathrm{Fe}_{6}$ (Fig. 9). The lifetime of $\mathrm{Fe}_{6} \mathrm{O}_{6}{ }^{+}$is $158 \pm 32 \mathrm{fs}$ and decreases by $\sim 54 \%$ with additional
Table 3. Fitting coefficients for the ultrafast transient dynamics of selected large $\mathrm{Fe}_{n} \mathrm{O}_{n+1}$ iron oxide clusters.

\begin{tabular}{lcccc} 
Species & $C_{E}$ & $\tau(\mathrm{fs})$ & $\mathrm{C}_{P}$ & $\nu$ \\
\hline $\mathrm{Fe}_{7} \mathrm{O}_{8}$ & 1.588 & $96 \pm 18$ & 0.116 & 0.073 \\
$\mathrm{Fe}_{8} \mathrm{O}_{9}$ & 1.454 & $139 \pm 22$ & 0.081 & 0.055 \\
$\mathrm{Fe}_{9} \mathrm{O}_{10}$ & 1.908 & $98 \pm 14$ & 0.126 & 0.066 \\
$\mathrm{Fe}_{10} \mathrm{O}_{11}$ & 1.694 & $107 \pm 15$ & 0.066 & 0.039 \\
$\mathrm{Fe}_{11} \mathrm{O}_{12}$ & 1.712 & $128 \pm 18$ & 0.092 & 0.054 \\
$\mathrm{Fe}_{12} \mathrm{O}_{13}$ & 1.564 & $120 \pm 15$ & 0.061 & 0.039 \\
$\mathrm{Fe}_{13} \mathrm{O}_{14}$ & 1.691 & $123 \pm 17$ & 0.070 & 0.041 \\
$\mathrm{Fe}_{14} \mathrm{O}_{15}$ & 2.022 & $103 \pm 18$ & 0.048 & 0.024 \\
$\mathrm{Fe}_{15} \mathrm{O}_{16}$ & 1.858 & $110 \pm 20$ & 0.021 & 0.011 \\
\hline
\end{tabular}

oxygen atoms. Interestingly, $\mathrm{Fe}_{6} \mathrm{O}_{7}{ }^{+}$has the shortest lifetime of all clusters detected in this study with a lifetime of only $68 \pm 10$ fs. Further, $\gamma$ decreases for $\mathrm{Fe}_{6} \mathrm{O}_{5}{ }^{+}$to $\mathrm{Fe}_{6} \mathrm{O}_{7}+$ from 0.130 to 0.049 with additional oxygen atoms, but $\mathrm{Fe}_{6} \mathrm{O}_{6}$ breaks the trend having a value of 0.163 .

\subsection{Larger Clusters}

For the $\mathrm{n}=7$ series, the addition of a single oxygen atom reduces the lifetime of the excited state by $\sim 38 \%$ from $133 \pm 13$ fs to $96 \pm 18$ fs (Fig. 10). This difference in lifetime between the two series is consistent with the trend obtained in the smaller clusters. For neutral $\mathrm{Fe}_{n} \mathrm{O}_{n+1}$ clusters, an excess oxygen atom has a binding energy exceeding $5 \mathrm{eV}$ and can exceed the binding energy of $\mathrm{FeO}$ units at these large cluster sizes. ${ }^{23}$ Therefore, the transient dynamics are recorded for the $(\mathrm{FeO})_{n}{ }^{+}$and $\mathrm{Fe}_{n} \mathrm{O}_{n+1}{ }^{+}$ series up to $n=12$ and 15 , respectively (supporting information, Fig. S3). The fitting coefficients for the larger $\mathrm{Fe}_{n} \mathrm{O}_{n+1}{ }^{+}$clusters are summarized in Table 3. For larger $\mathrm{Fe}_{n} \mathrm{O}_{n+1}{ }^{+}$clusters, the fs lifetimes are comparable to the similar sized $(\mathrm{FeO})_{n}$ clusters. Small fluctuations are recorded in the fs lifetime of both series with changes in size, but remain consistent within the error bars to be an average of $115 \mathrm{fs}$. This suggests that the influence of

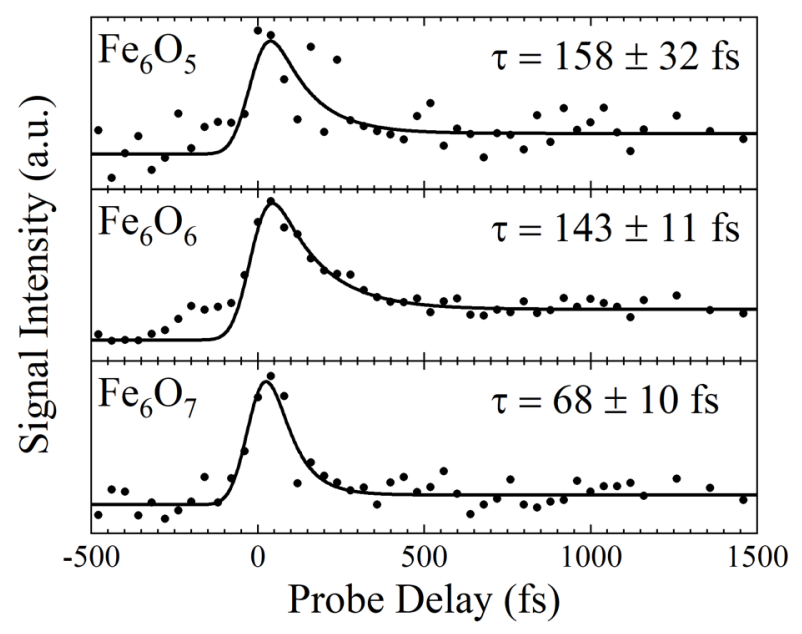

Fig. 9 Pump probe signal of the $\mathrm{Fe}_{6} \mathrm{O}_{m}{ }^{+}(m=5-7)$ series. 


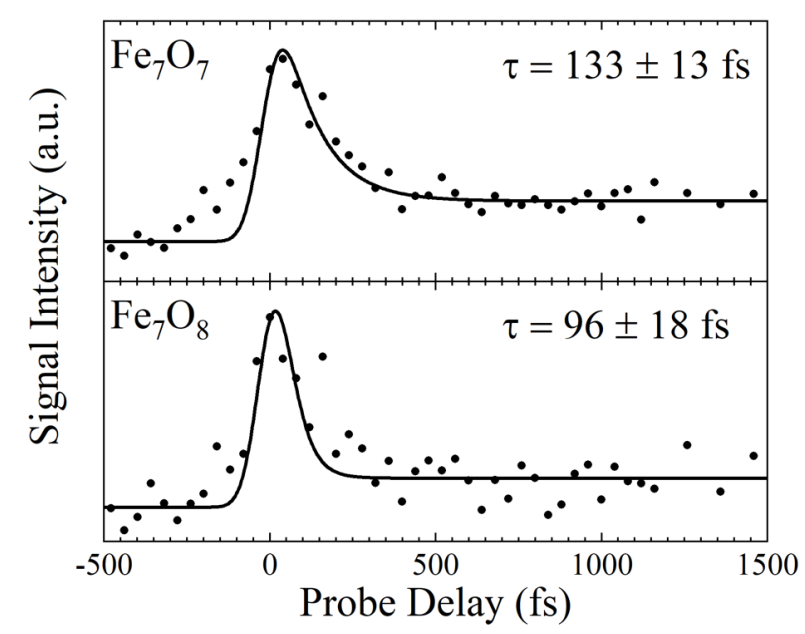

Fig. 10 Pump probe signal of the $\mathrm{Fe}_{7} \mathrm{O}_{m}{ }^{+}(m=7-8)$ series.

the additional oxygen atom is small for these larger clusters. The small increase in overall oxidation state does not have a significant influence on the transient dynamics and the lifetimes of the two cluster series are similar. According to IVR, the fast component of the $\mathrm{Fe}_{n} \mathrm{O}_{n+1}{ }^{+}$clusters should decrease with size because of the larger degrees of freedom to dissipate the energy. However, as described earlier, these clusters have already exceeded the size where a saturation in the relaxation dynamics was achieved.

The long-lived excited states remain accessible in larger clusters for both cluster series. For $\mathrm{n}=8$, the bound state accounts for $\sim 10 \%$ of the total population and decreases linearly with the addition of each FeO unit. This is consistent with the fact that larger clusters contain a larger density of states than smaller clusters, thereby enabling the electronic energy conversion into vibrational motion while returning to the ground electronic state.

\section{Conclusions}

The ultrafast ionization and electronic relaxation dynamics of neutral finite-sized iron oxide clusters have been studied using two color pump-probe fs spectroscopy. With fs ionization, the stoichiometric clusters, $(\mathrm{FeO})_{n}$, dominate the cluster distribution for $\mathrm{n}<10$ and $\mathrm{Fe}_{n} \mathrm{O}_{n+1}$ becomes dominant for values of $n>10$. In our experiment, weakly bound oxygen rich clusters are not observed with fs laser pulses, suggesting that the clusters detected in our mass spectra are stable to within the energy of 1 probe photon. Although fragmentation is possible, it was not observed with the exception of $\mathrm{Fe}_{2}$. The dissociation pathways cannot be determined from these studies and are a subject of future experiments.

Upon irradiation of a $400 \mathrm{~nm}$ fs pulse, an electron migrates from the $\mathrm{O}$ atom to the non-bonding orbitals of the $\mathrm{Fe}$ atom within the cluster. This results in a bifurcation of the initial wavepacket with a large proportion of the wavepacket relaxing on the fs timescale to a low-lying electronic state and is assisted by IVR. A smaller fraction of the wavepacket remains in a long- lived ( 20 ps) bound state where the excited electron remains in the nonbonding Fe orbitals.

Transient signals of the neutral iron oxide clusters are compared as a function of growing cluster size and stoichiometric composition. The lifetime of the excited states of the clusters decreases rapidly from $\sim 350$ fs to $\sim 140$ fs until the clusters adopt a 3D stoichiometry, then remain roughly independent of cluster size. Changes in the oxidation state of the Fe atoms has a large effect on the lifetime of small clusters, where higher oxidation states have shorter lifetimes. The shortest lifetimes are obtained for mid-range clusters with an extra oxygen $\left(\mathrm{Fe}_{6} \mathrm{O}_{7}\right.$ and $\left.\mathrm{Fe}_{7} \mathrm{O}_{8}\right)$, which are among the smallest clusters recorded that contain an excess oxygen atom. As the cluster size grows, a smaller proportion of the wavepacket remains in the long-lived bound excited state.

\section{Conflicts of interest}

There are no conflicts to declare.

\section{Acknowledgements}

We gratefully acknowledge support from ASU Light-Works, Sustainable Fuels and Products. J.G also acknowledges support from Western Alliance to Expand Student Opportunities (WAESO) Louis Stokes Alliance for Minority Participation (LSAMP) Bridge to Doctorate (BD) National Science Foundation (NSF) Grant No. HRD-1702083.

1 R. Schlögl, Angew. Chemie Int. Ed., 2003, 42, 2004-2008.

2 C. Rhodes, G. J. Hutchings and A. M. Ward, Catal. Today, 1995, 23, 43-58.

3 P. C. Frisch and J. D. Slavin, Earth Planets Sp., 2013, 65, 175-182.

4 I. McDonald, G. C. Sloan, A. A. Zijlstra, N. Matsunaga, M. Matsuura, K. E. Kraemer, J. Bernard-Salas and A. J. Markwick, Astrophys. J. Lett., 2010, 717, L92-7.

5 X. K. Zhu, Y. Guo, R. K. O'Nions, E. D. Young and R. D. Ash, Nature, 2001, 412, 311-313.

6 A. Li, J. M. Liu and B. W. Jiang, Astrophys. J., 2013, 777, 1-7.

7 N. M. Reilly, J. U. Reveles, G. E. Johnson, J. M. Del Campo, S. N. Khanna, A. M. Köster and A. W. Castleman Jr., J. Phys. Chem. C, 2007, 111, 19086-19097.

8 N. M. Reilly, J. U. Reveles, G. E. Johnson, S. N. Khanna and A. W. Castleman Jr., Chem. Phys. Lett., 2007, 435, 295-300.

9 N. M. Reilly, J. Ulises Reveles, G. E. Johnson, S. N. Khanna and A. W. Castleman Jr., J. Phys. Chem. A, 2007, 111, 4158-4166.

10 D. Schröder, H. Schwarz, D. E. Clemmer, Y. Chen, P. B. Armentrout, V. I. Baranov and D. K. Böhme, Int. J. Mass Spectrom. Ion Process., 1997, 161, 175-191.

11 D. Schröder, R. Wesendrup, C. A. Schalley, W. Zummack and H. Schwarz, Helv. Chim. Acta, 1996, 79, 123-132.

12 M. C. Oliveira, J. Marçalo, M. C. Vieira and M. A. A. Ferreiraac, Int. J. Mass Spectrom., 1999, 185, 825-835.

13 T. Yumura, T. Amenomori, Y. Kagawa and K. Yoshizawa, J. Phys. Chem. A, 2002, 106, 621-630.

14 W. Xue, Z. C. Wang, S. G. He, Y. Xie and E. R. Bernstein, J. Am. 
Chem. Soc., 2008, 130, 15879-15888.

15 Y. Xie, F. Dong, S. Heinbuch, J. J. Rocca and E. R. Bernstein, J. Chem. Phys., 2009, 130, 114306-11.

16 S. Y. Lv, Q. Y. Liu, J. J. Chen and S. G. He, J. Phys. Chem. C, 2019, 123, 25949-25956.

17 S. Yin, W. Xue, X. L. Ding, W. G. Wang, S. G. He and M. F. Ge, Int. J. Mass Spectrom., 2009, 281, 72-78.

18 B. Maunit, A. Hachimi, P. Manuelli, P. J. Calba and J. F. Muller, Int. J. Mass Spectrom. Ion Process., 1996, 156, 173-187.

19 B. Yan, L. Li, Q. Yu, W. Hang, J. He and B. Huang, J. Am. Soc. Mass Spectrom., 2010, 21, 1227-1234.

20 M. Sakurai, K. Sumiyama, Q. Sun and Y. Kawazoe, J. Phys. Soc. Japan, 1999, 68, 3497-3499.

21 D. N. Shin, Y. Matsuda and E. R. Bernstein, J. Chem. Phys., 2004, 120, 4157-4164.

22 D. N. Shin, Y. Matsuda and E. R. Bernstein, J. Chem. Phys., 2004, 120, 4150-4156.

23 N. O. Jones, B. V. Reddy, F. Rasouli and S. N. Khanna, Phys. Rev. B - Condens. Matter Mater. Phys., 2005, 72, 165411-165414.

24 M. Ju, J. Lv, X. Y. Kuang, L. P. Ding, C. Lu, J. J. Wang, Y. Y. Jin and G. Maroulis, RSC Adv., 2015, 5, 6560-6570.

25 A. Erlebach, C. Hühn, R. Jana and M. Sierka, Phys. Chem. Chem. Phys., 2014, 16, 26421-26426.

26 G. L. Gutsev, K. G. Belay, K. V. Bozhenko, L. G. Gutsev and B. R. Ramachandran, Phys. Chem. Chem. Phys., 2016, 18, 2785827867.

27 K. Ohshimo, T. Komukai, R. Moriyama and F. Misaizu, J. Phys. Chem. A, 2014, 118, 3899-3905.

28 G. V. Chertihin, W. Saffel, J. T. Yustein, L. Andrews, M. Neurock, A. Ricca and C. W. Bauschlicher, J. Phys. Chem., 1996, 100, 52615273.

29 L. Andrews, G. V. Chertihin, A. Ricca and C. W. Bauschlicher, J. Am. Chem. Soc., 1996, 118, 467-470.

30 A. Kirilyuk, A. Fielicke, K. Demyk, G. Von Helden, G. Meijer and T. Rasing, Phys. Rev. B - Condens. Matter Mater. Phys., 2010, 82, 1013.

31 M. Krauss and W. J. Stevens, J. Chem. Phys., 1985, 82, 5584-5596.

32 C. N. Sakellaris, E. Miliordos and A. Mavridis, J. Chem. Phys., 2011, 134, 234308-16.

33 M. F. A. Hendrickx and K. R. Anam, J. Phys. Chem. A, 2009, 113, 8746-8753.

34 D. A. Chestakov, D. H. Parker and A. V. Baklanov, J. Chem. Phys., 2005, 122, 1-4.

35 J. Fan and L. Wang, J. Chem. Phys., 1995, 102, 8714-8717.

36 P. C. Engelking and W. C. Lineberger, J. Chem. Phys., 1977, 66, 5054-5058.

37 G. L. Gutsev, C. W. Bauschlicher, H. J. Zhai and L. S. Wang, J. Chem. Phys., 2003, 119, 11135-11145.

38 L. S. Wang, H. Wu and S. R. Desai, Phys. Rev. Lett., 1996, 76, 48534856.

39 H. Wu, S. R. Desai and L. S. Wang, J. Am. Chem. Soc., 1996, 118, 5296-5301.

40 M. Velegrakis, C. Mihesan and M. Jadraque, J. Phys. Chem. A, 2013, 117, 2891-2898.

41 P. Armentrout, Annu. Rev. Phys. chem., 2001, 52, 423-461.

42 D. Schröder, P. Jackson and H. Schwarz, Eur. J. Inorg. Chem, 2000, 2000, 1171-1175.
43 M. Li, S. R. Liu and P. B. Armentrout, J. Chem. Phys., 2009, 131, 144310-16.

44 K. Koyama, S. Kudoh, K. Miyajima and F. Mafuné, Chem. Phys. Lett., 2015, 625, 104-109.

45 K. S. Molek, C. Anfuso-Cleary and M. A. Duncan, J. Phys. Chem. A, 2008, 112, 9238-9247.

46 G. L. Gutsev, K. G. Belay, L. G. Gutsev and B. R. Ramachandran, Comput. Mater. Sci., 2017, 137, 134-143.

47 W. C. Wiley and I. H. McLaren, Rev. Sci. Instrum., 1955, 26, 11501157.

48 S. Pedersen and A. H. Zewail, Mol. Phys., 1996, 89, 1455-1502.

49 R. B. Metz, C. Nicolas, M. Ahmed and S. R. Leone, J. Chem. Phys., 2005, 123, 114313.

50 D. L. Hildenbrand, Chem. Phys. Lett., 1975, 34, 352-354. 